\title{
Genotypes and phenotypes of resistance in Ecuadorian Plasmodium falciparum
}

\author{
Gabriela Valenzuela' ${ }^{1}$ L. Enrique Castro², Julio Valencia-Zamora², Claudia A. Vera-Arias ${ }^{1}$, Petra Rohrbach ${ }^{3}$ \\ and Fabián E. Sáenz ${ }^{1 *}$ (])
}

\begin{abstract}
Background: Malaria continues to be endemic in the coast and Amazon regions of Ecuador. Clarifying current Plasmodium falciparum resistance in the country will support malaria elimination efforts. In this study, Ecuadorian $P$. falciparum parasites were analysed to determine their drug resistance genotypes and phenotypes.

Methods: Molecular analyses were performed to search for mutations in known resistance markers (Pfcrt, Pfdhfr, Pfdhps, Pfmdr1, k13). Pfmdr1 copy number was determined by qPCR. PFMDR1 transporter activity was characterized in live parasites using live cell imaging in combination with the Fluo-4 transport assay. Chloroquine, quinine, lumefantrine, mefloquine, dihydroartemisinin, and artemether sensitivities were measured by in vitro assays.

Results: The majority of samples from this study presented the CVMNT genotype for Pfcrt (72-26), NEDF SDFD mutations in Pfmdr1 and wild type genotypes for Pfdhfr, Pfdhps and k13. The Ecuadorian P. falciparum strain ESM-2013 showed in vitro resistance to chloroquine, but sensitivity to quinine, lumefantrine, mefloquine, dihydroartemisinin and artemether. In addition, transport of the fluorochrome Fluo-4 from the cytosol into the digestive vacuole (DV) of the ESM-2013 strain was minimally detected in the DV. All analysed samples revealed one copy of Pfmdr1.

Conclusion: This study indicates that Ecuadorian parasites presented the genotype and phenotype for chloroquine resistance and were found to be sensitive to $\mathrm{SP}$, artemether-lumefantrine, quinine, mefloquine, and dihydroartemisinin. The results suggest that the current malaria treatment employed in the country remains effective. This study clarifies the status of anti-malarial resistance in Ecuador and informs the P. falciparum elimination campaigns in the country.
\end{abstract}

Keywords: Plasmodium falciparum, Malaria, Ecuador, Resistance, Genotypes, Phenotypes

\section{Background}

According to World Health Organization (WHO), 132 million people were at risk of malaria infection in 2015 in the Americas. Between 2010 and 2015 there was an estimated $31 \%$ decrease in malaria incidence in this region, as well as a $37 \%$ decrease in malaria-related mortality. Nevertheless, approximately 450,000 cases were reported in 2015 in this region [1], 30\% from Venezuela, 11\% from Colombia and 15\% from Peru [1]. Thirty per cent

\footnotetext{
*Correspondence: fsaenz213@puce.edu.ec

${ }^{1}$ Centro de Investigación para la Salud en América Latina, Facultad de Ciencias Exactas y Naturales, Pontificia Universidad Católica del Ecuador, Av. 12 de Octubre 1076, Apartado, 17-01-2184 Quito, Ecuador

Full list of author information is available at the end of the article
}

of all cases were caused by Plasmodium falciparum. Even though, Ecuador accounted for less than $1 \%$ of the region malaria cases in 2015, malaria continues to be endemic in the coast and Amazon areas of Ecuador. From 2016 to 2018, Ecuador has seen increases in malaria cases (1191 in 2016, 1380 in 2017 and 1806 in 2018), where P. falciparum was responsible for $10 \%$ of these cases [2].

The current treatment for $P$. falciparum infection in the Americas is based on artemisinin in combination with another anti-malarial (ACT) [3]. Treatment for uncomplicated falciparum malaria in Ecuador relies on the combination artemether-lumefantrine + primaquine; for Plasmodium vivax the treatment used is chloroquine + primaquine [4]. Plasmodium falciparum has developed resistance to almost all available 
anti-malarials, necessitating the need for an adequate knowledge of anti-malarial drug effectiveness. This is especially true in low transmission areas, where malaria elimination is ongoing, as the inflow of resistant parasites can generate unwanted outbreaks.

Chloroquine (CQ) resistance in P. falciparum was reported in 1957 on the Thailand-Cambodian border in Southeast Asia and almost at the same time in Colombia and Venezuela in South America, before spreading to the rest of the world [5]. Mutations in the P. falciparum chloroquine resistance transporter (PfCRT) are considered the main reason for CQ resistance [6]. Currently, CQ resistance is found throughout South America [5] and the PfCRT molecular marker K76T, thought to be mainly responsible for CQ resistance, is considered fixed in this region [7]. The PfCRT haplotype CVMNT (positions 72-76) has been reported in Colombia and Peru, while CVMET and CVEIT have been reported in Colombia and Venezuela and SVMNT has been reported in the Amazon region of Brazil and Peru [8].

During the 1970s the combination sulfadoxinepyrimethamine (SP) was introduced in South America as treatment against $P$. falciparum. Shortly after the introduction, resistance to these drugs was reported [8]. Colombian, Brazilian and Peruvian parasite isolates showed mutations in Pfdhps mainly in positions 437, 540 and 581. The mutation A437G is dominant in Colombia, while the mutations A437G and K540E are found in Peru [7-10]. In addition, Venezuela and Bolivia have reported the mutation $\mathrm{K} 540 \mathrm{E}$ in $90 \%$ of the parasite samples tested [7, 8]. Pfdhfr mutations C50R, I165L and S108N/T are common throughout South America $[8,9]$ and all mutations are associated with SP resistance [11].

Plasmodium falciparum multidrug resistance 1 (Pfmdr1) transporter gene encodes for a p-glycoprotein that is part of the adenosine triphosphate-binding cassette transporter family. Mutations in Pfmdr1 are associated with multidrug resistance, and show reduced susceptibility to mefloquine (MQ), halofantrine (HF), quinine (QN), and possibly lumefantrine (LUMF) [11, 12]. The PfMDR1 mutations N86Y and Y184F are common in Asia and Africa, while the mutations S1034C, N1042D and D1246Y are mostly found in South America [13].

Several studies associate increases in $P f m d r 1$ copy number to MQ resistance, and QN and CQ susceptibility [13-16]. Recent research suggested that an increase in Pfmdr1 copy number is related to artemisinin resistance $[13,14]$. In South America, there are reports of changes in Pfmdr1 copy number, specifically in samples coming from the Pacific region, Atlantic region and southeastern Colombia, where an increase of 2 to 5 copies of $P f m d r 1$ were found in $30 \%$ of the parasite samples [15]. Peru reported single Pfmdr1 copy numbers [17].

Resistance to artemisinin (ART) in P. falciparum has been reported in five Asian countries: China, Vietnam, Cambodia, Thailand, and Myanmar. The current management to control $P$. falciparum infections is based on ART derivatives combined with a partner anti-malarial (e.g., MQ, LUMF, primaquine) [18]. Kelch 13 (k13) propeller mutations have been associated with ART resistance and can be used as molecular markers to monitor the possible emergence of ART resistance $[3,18]$. ACT treatment continues to be effective in South America. New studies in Brazilian, Peruvian and Colombian isolates show no $k 13$ mutations associated with ART resistance $[5,19,20]$.

In addition to genetic variability studies, drug resistant phenotypes can be characterized using in vitro assays. In particular, Colombia reported low in vitro susceptibility to CQ and amodiaquine (AQ) in almost $90 \%$ of the isolates analysed, showing $\mathrm{IC}_{50}$ values for both anti-malarials greater than $100 \mathrm{nM}[14,21]$. Furthermore, all samples showed high susceptibility to dihydroartemisinin (DHA), LUMF and artemether (ATM) [14, 21, 22]. Brazilian samples from the Amazon region also showed resistance to $C Q$ and $A Q$, with an elevated $\mathrm{IC}_{50}$ [23]. In vitro assays with field parasites in South America have been limited, since culture adaptation of field parasites to laboratory conditions require a long time and are usually challenging [24].

In 2002, studies from Ecuador reported mutations in isolates collected in Esmeraldas. The parasites presented the CVMNT Pfcrt genotype and one, (position $108 \mathrm{~N}$ ) two (positions 108N, 164L) or three (51I, 108N, 164L) mutations in Pfdhfr [25]. In 2013, parasite isolates showed wild type genotypes for Pfdhfr and Pfdhps, the CVMNT and CVMET Pfcrt genotypes, and the mutations Y184F and N1042D in Pfmdr1 in an outbreak that occurred in Esmeraldas [26]. These genotypes indicated that Ecuadorian strains were CQ resistant and mostly sensitive to sulfadoxine and pyrimethamine [26]. This genotype was shared with Ecu 1110, a 1990 isolate from the same area. Ecu1110 has an in vitro CQ resistance phenotype $\left(\mathrm{IC}_{50}>90 \mathrm{nM}\right)$ [27].

In this study, in vitro assays were used to determine drug susceptibility phenotypes. In addition, drug resistance genotypes were analysed in Pfcrt, Pfdhfr, Pfdhps, pfmdrl, and $k 13$ of Ecuadorian P. falciparum isolates. The aim of this study was to understand current antimalarial resistance in Ecuador, in order to support malaria elimination efforts in the country. 


\section{Methods}

\section{Ethics statement}

The samples used in this study were obtained by the malaria control and elimination programme and approved by the Ethical Review Committee of Pontificia Universidad Católica del Ecuador (CBE-016-2013 and CEISH-163-2016). Written informed consent was provided by study participants and/or their legal guardians.

\section{Study site and sample collection}

Sixty-nine samples were analysed in this study, 62 of these (89.9\%) were collected in Esmeraldas province. Esmeraldas is located in northwest Ecuador and borders with Colombia. All the samples collected in this province came from Esmeraldas and San Lorenzo counties, where the incidence of $P$. falciparum is the highest in Ecuador. Four samples were collected in the Carchi province, located in the north of Ecuador, east of the Esmeraldas province. Three samples were collected in Sucumbíos, a province located in the north Amazon of Ecuador, east of Carchi province (Fig. 1). All samples were collected between 2013 and 2015 through the National Service for Control of Diseases Transmitted by Arthropod Vectors (SNEM). Eighty-five per cent of the samples were collected as whole blood and $15 \%$ on filter paper and kept at $4{ }^{\circ} \mathrm{C}$.

\section{DNA extraction and infection confirmation}

The DNA from 69 samples was isolated from filter paper using QIamp DNA mini-spin kit (QIAGEN, Valencia, CA, USA), and from whole blood using Axyprep body fluid viral DNA/RNA Miniprep (AXYGEN, Union City, CA, USA). Confirmation of $P$. falciparum infection was performed by microscopy (slides were stained with Giemsa stain 20\%, for $20 \mathrm{~min}$ ) [28] and nested-PCR using the $18 \mathrm{~S}$ ribosomal RNA gene. Genomic DNA was used for Plasmodium spp. detection. DNA was amplified using
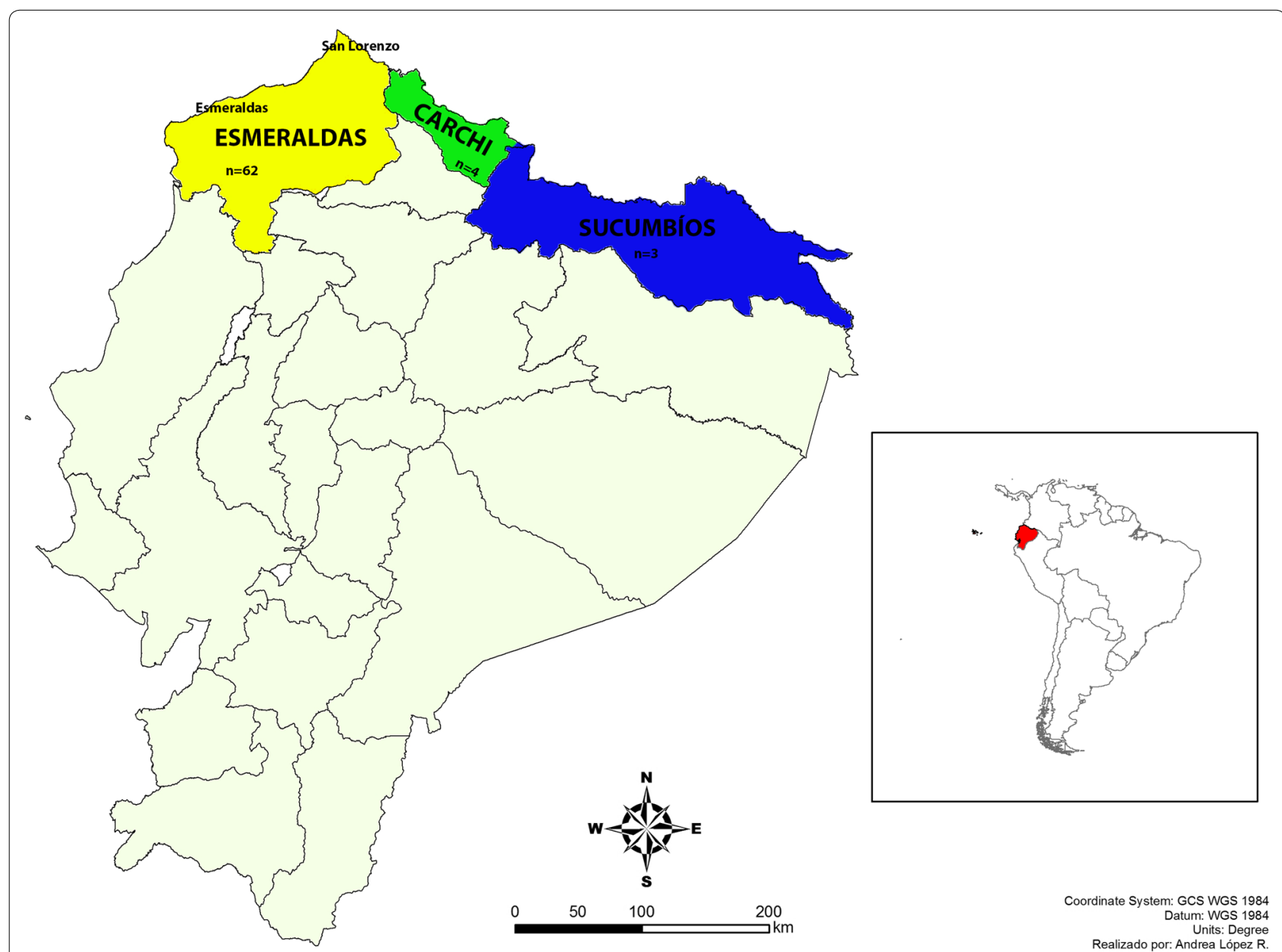

Fig. 1 Study site. All samples were collected in three northern provinces of Ecuador: Esmeraldas $n=62$ samples, Carchi $n=4$ samples and Sucumbíos $n=3$ samples 
nested PCR as previously described [29]. The amplification was done using primers that target the $18 \mathrm{~S}$ ribosomal of Plasmodium spp. The $25 \mu \mathrm{l}$ reaction contained $2.5 \mu \mathrm{l}$ of $\mathrm{Mg} 2 \mathrm{Cl}, 3 \mu \mathrm{l}$ of water, $12.5 \mu \mathrm{l}$ of Green GoTaq Master Mix, $1 \mu \mathrm{l}$ of each primer and $5 \mu \mathrm{l}$ of DNA [29].

\section{Analysis of drug resistance markers}

Plasmodium falciparum 3D7, W2, D6, CAM6 and C2B isolates were used as controls and Ecuadorian P. falciparum were analysed to identify drug resistance-associated mutations in Pfcrt (positions 72-76), Pfmdr1 (positions 134, 184, 1034, 1042, 1226, 1246), Pfdhfr (positions 51, 59, 108, 164), Pfdhps (positions 436, 437, 540, 581, 613), and $k 13$ (positions $476,493,539,543,580$ ) using conditions and primers reported previously in other studies $[9$, 27-29] (Additional file 1: Table S1). All PCR amplicons were visualized on $2 \%$ agarose gels and purified with $5 \mu \mathrm{l}$ of Illustra ExoProStar (GE Healthcare, Piscataway NJ, USA) at $37^{\circ} \mathrm{C}$ for $25 \mathrm{~min}$ and $20 \mathrm{~min}$ at $80^{\circ} \mathrm{C}$. After visual confirmation of amplified product, all amplicons were submitted to MACROGEN, South Korea, for capillary sequencing. Sanger sequencing was performed on all samples to identify drug resistance-associated mutations in $p f c r t, p f d h f r, p f d h p s, p f m d r 1$ and $k 13$ genes.

The sequencing results were analysed and aligned using Geneious software version 10 (Biomatters, Inc, Newark, NJ, USA). The sequences were compared to controls (3D7, W2, D6, CAM6, C2B) to establish which samples present mutations. The sequence alignments were analysed, and SNP differences were established to determine the mutation frequencies for each gene. All mutations frequencies were analysed using Microsoft Excel 16.9.

\section{Pfmdr1 copy number}

Pfmdr1 copy number was established with real time PCR, using TaqMan probes for Pfmdrl (target gene), and the housekeeping gene Seryl-t-rna-synthetase was used as a control.

The primers used for quantifying copy number were: $P F_{-} F:$ 5TTAAGTTTTACTCTAAAAGAAGGG AAAACATA,PF_ $R: \quad 5$ 'TCTCCTTCGGTTGGATCA TAAAG, seryl_F:5'GATTTATTAAGAAAAATAGGT GGAGCTA, seryl_R:5'TATAGCATTATGTAATAA GAAACCTGC, and the Taqman probes were: $P F_{-}$ FAM: 5'FAMCATTTGTGGGAGAATCAGGTTGTG GGAAAT_TAMRA, seryl_probe:5'VICAAGGTATA CAAGTAGCAGGTCATCGTGGTT_TAMRA [30].

Each $20 \mu \mathrm{l}$ of reaction mix contained $15 \mathrm{ng}$ DNA, $300 \mathrm{nM}$ primers, $250 \mathrm{nM}$ TaqMan probes and $10 \mu \mathrm{l}$ TaqMan Fast Advance Mix from Applied Biosystems (Austin, TX, USA). Triplicates of each sample were analysed using the following amplification protocol initial activation: $94{ }^{\circ} \mathrm{C}$ for $3 \mathrm{~min}$, followed by 40 cycles of: $94{ }^{\circ} \mathrm{C}$ for $1 \mathrm{~min}, 60{ }^{\circ} \mathrm{C}$ for $1 \mathrm{~min}$, and $72{ }^{\circ} \mathrm{C}$ for $30 \mathrm{~s}$. Fluorescence was recorded after each elongation step. Real-time PCR was carried out in StepOne ${ }^{\mathrm{TM}}$ Real-Time PCR System (Applied Biosystems). Copy numbers were calculated relative to $3 \mathrm{D} 7$, which is known to have only one Pfmdr1 gene copy; the seryl gene is consistently expressed throughout the parasite blood stages. The copy number of $P f m d r 1$ was calculated using relative quantification (RQ) by the calculation of $\Delta \Delta \mathrm{Ct}$, using the $\mathrm{Ct}$ generated by Seryl and $P f m d r 1$ for each sample by a comparative threshold method, with the formula $\Delta \Delta \mathrm{Ct}=(\mathrm{Ct}$ $\left.m d r 1-\mathrm{Ct}_{\text {seryl }}\right)_{\text {Sample }}-\left(\mathrm{Ct}_{m d r 1}-\mathrm{Ct}_{\text {seryl }}\right)_{3 \mathrm{D} 7}, 2^{-(\Delta \Delta \mathrm{Ct})}$ where $\mathrm{Ct}$ is the threshold cycle for each gene [16].

\section{Phenotype characterization \\ Parasite strains and culture conditions}

The standard strains 3D7 (CQ sensitive) and W2 (CQ and SP resistant) were used as controls for in vitro susceptibility assays: P. falciparum isolate ESM-2013 was obtained from a patient in Esmeraldas city in 2013 and subsequently adapted to laboratory conditions. Parasites were cultured in human $\mathrm{O}+$ red blood cells, following previously reported methods [31], with modifications. RPMI 1640, supplemented with 25 mM HEPES, $8,9 \mathrm{mM}$ sodium bicarbonate and human $\mathrm{O}+$ plasma was used as complete medium. All parasites were cultured in red blood cells at $4 \%$ haematocrit with complete medium at $37{ }^{\circ} \mathrm{C}$ and mixed gas $\left(5 \% \mathrm{O}_{2}, 5 \% \mathrm{CO}_{2}\right.$ and $90 \%$ of $\mathrm{N}_{2}$ or $3 \% \mathrm{O}_{2}, 5 \% \mathrm{CO}_{2}$ and $92 \% \mathrm{~N}_{2}$ according to laboratory conditions).

\section{Drug sensitivity assay}

In vitro sensitivity assays were performed with the following drugs: chloroquine disphosphate (CQ), dihydroartemisinin (DHA), quinine sulfate $(\mathrm{QN})$, mefloquine hydrochloride (MQ), lumefantrine (LUMF) and artemether (ATM). The drugs were provided by Dennis Kyle, University of South Florida. Drug sensitivity in vitro assays of the parasites was performed by microscopy to establish the $\mathrm{IC}_{50} \mathrm{~s}$ of several anti-malarial drugs. Drug stock solutions were prepared in dimethyl sulfoxide (DMSO) or water, at an initial concentration of $1 \mathrm{mg} /$ $\mathrm{ml}$. The experiments were set up in 96-well plates with 2 -fold dilutions of each drug across the plate in a total volume of $150 \mu \mathrm{l}$ and at a final red blood cell concentration of $1.5 \%$ ( $\mathrm{vol} / \mathrm{vol})$. The experiment was started at an initial parasitaemia of $0.5 \%$ (80\% rings) synchronous parasite-infected red blood cells (PRBC). The plates were incubated for $72 \mathrm{~h}$ at $37^{\circ} \mathrm{C}$ in an atmosphere of $5 \% \mathrm{CO}_{2}$, $5 \% \mathrm{O}_{2}$, and $90 \% \mathrm{~N}_{2}$. A light microscope was used to look for the presence of schizonts in a thick smear to establish parasite growth. 
Parasitaemia was determined by counting 1000 red blood cells (RBCs) in thin smears and the number of infected cells (iRBCs) using the equation (No. iRBCs*100/No. RBCs). All in vitro assays were performed in duplicate with at least two replicates.

Thin blood smears were fixed in methanol and stained with $20 \%$ GIEMSA for $20 \mathrm{~min}$. The data were analysed using Excel 16.9 software to compare the parasitaemia of each well versus drug concentration. Parasite $\mathrm{IC}_{50}$ curves were obtained using non-linear regression and $\mathrm{IC}_{50}$ s were calculated by using the equation of the curve. Linear point interception with the curve was used to establish inhibitory concentrations.

\section{Dye loading and live cell imaging}

The laboratory strain Dd2 and the Ecuadorian P. falciparum isolate ESM-2013 were used for this experiment. Synchronized trophozoite stage parasites were loaded with $5 \mu \mathrm{M}$ Fluo-4 AM (Life Technologies, Burlington, $\mathrm{ON}$, Canada) in Ringer's solution (122.5 mM NaCl, $5.4 \mathrm{mM} \mathrm{KCl}, 1.2 \mathrm{mM} \mathrm{CaCl}_{2}, 0.8 \mathrm{mM} \mathrm{MgCl}_{2}, 11 \mathrm{mM}$ D-glucose, $10 \mathrm{mM}$ HEPES, $1 \mathrm{mM} \mathrm{NaH} \mathrm{PO}_{4}, \mathrm{pH}$ 7.4) for $50 \mathrm{~min}$ at $37{ }^{\circ} \mathrm{C}$. Parasites were washed twice with Ringer's solution and transferred to a microscope chamber, where they were kept at $37{ }^{\circ} \mathrm{C}$ during microscopy. A series of images per parasite was taken using a Zeiss LSM710 confocal microscope (Carl Zeiss, Oberkochen, Germany) equipped with a water-corrected objective (C-apochromat $63 \times / 1.20 \mathrm{~W}$ Korr M27) and a $488 \mathrm{~nm}$ laser $(12.5 \mathrm{~mW}, 2 \%$ intensity). The range of emitted fluorescence was measured from 493 to $622 \mathrm{~nm}$. The acquired images were analysed using the Zen software (Carl Zeiss) and the fluorescence averaged [32].

\section{Results}

\section{Genotyping of drug resistance markers}

Sixty-two out of the 69 collected samples were analysed for mutations in five genes: Pfcrt (positions 72-76), Pfdhps (positions 436, 437, 540, 581, 613), Pfdrfr (positions 50, 51, 59, 108, 164), Pfmdr1 (positions 86, 130, $144,184,1034,1042,1226,1246$ ) and $k 13$ (positions 476, 493, 539, 543, 580). Thirty-two samples from Esmeraldas county and 23 samples from San Lorenzo county (total of 55 samples in Esmeraldas province) were collected, as well as 4 samples in Carchi province and 3 in Sucumbíos province. F50 is a sample collected in Nuevo Rocafuerte, Aguarico, Orellana province in 2014; this province is located on the east border of Ecuador to Peru. In addition, Ecu 1110 was collected in Esmeraldas in 1990, this province is located in northern border and was included in the analysis.

The samples collected in Esmeraldas county presented two mutated haplotypes of Pfcrt: $97 \%$ of the samples presented the CVMNT haplotype with a simple mutation in the position $76(\mathrm{~K} 76 \mathrm{~T})$ and $3 \%$ of the samples showed the CVMET haplotype with double mutations at positions 75 (N75E) and 76. Thirty-one of these samples were reported in a previous study [26]. In San Lorenzo county, the same haplotypes were found but in different frequencies, $78.3 \%$ of the samples presented the haplotype CVMNT and 21.3\% showed CVMET. All of the samples collected in Carchi showed the CVMNT haplotype. This haplotype occurs in $33.3 \%$ of Sucumbíos samples, while, $66.7 \%$ presented the CVMET haplotype (Fig. 2a). Only the sample F50 collected in Orellana presented the haplotype SVMNT with double mutations at positions 72 and 76 (C72S). The Ecu1110 isolate, collected in Esmeraldas in 1990, also presented the CVMNT haplotype for Pfcrt. In summary, $100 \%$ of the samples analysed showed at least one mutation in the $72-76$ positions of Pfcrt. All of the genotypes found are associated to CQ resistance (Table 1).

Pfdhfr presented the wild genotype CNCSI in the majority of samples: $97 \%$ in Esmeraldas county, $82.6 \%$ in San Lorenzo county, 66.7\% in Sucumbíos and 100\% in Carchi. The CNCNI haplotype, with a simple mutation in the position S108N, was found in $13.04 \%$ of San Lorenzo samples, and the CICNI haplotype (double mutant at positions N51I and S108N) in 4.36\% of San Lorenzo samples and 33\% of Sucumbíos samples (Fig. 2b). The double mutation (CICNI) was also found in F50 (Orellana). In contrast, Ecu 1110 presented the wild type genotype CNCSI. San Lorenzo county showed higher diversity of haplotypes for the Pfdhfr gene (Table 1), although, the wild type genotype was the most frequent in the population.

The wild type genotype (SAKAA) of Pfdhps was the dominant genotype in all samples analysed: $97 \%$ of Esmeraldas samples and $100 \%$ of San Lorenzo, Carchi and Sucumbíos samples (Fig. 2c). One sample from Esmeraldas county presented a synonymous mutation in the position 540, the sample F50 from Sucumbíos had the mutation $\mathrm{K} 540 \mathrm{E}$, showing the haplotype SAEAA (Table 1).

Mutations in the gene $k 13$ have been related to artemisinin sensitization. This gene was analysed in five separate positions $(476,493,539,543$, and 580). All the samples studied in Esmeraldas, San Lorenzo, Carchi and Sucumbíos presented the wild type haplotype CRYGI (Fig. 2d).

Pfmdr1 presented two main mutations: Y184F and N1042D in the majority of samples analysed in this study. In fact, the NEDFSDFD haplotype was present in $97 \%$ of Esmeraldas county samples, $78.3 \%$ of San Lorenzo county samples and $100 \%$ of Carchi and Sucumbíos samples (Fig. 2e). Surprisingly, the F50 sample and the Ecu 1110 

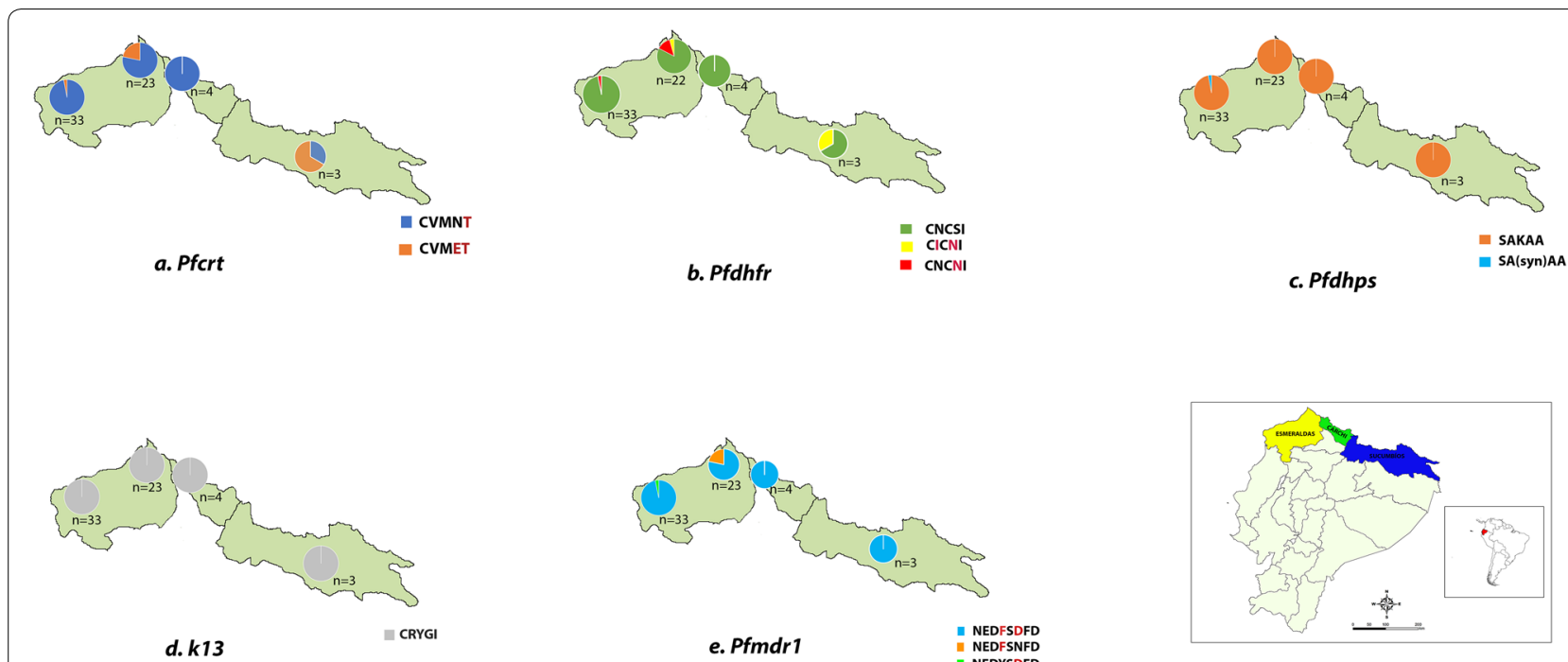

Fig. 2 Drug resistance haplotype distribution and frequency in Ecuadorian Plasmodium falciparum study area. Distribution of a Pfcrt, b Pfdhfr, c Pfdhps, d k13, e Pfmdr 1 haplotypes

also presented the genotype NEDFSDFD. In contrast, only 3\% of Esmeraldas county (F31 sample) presented the mutation 1042 (NEDYSDFD) alone, and 21.7\% of San Lorenzo county samples presented the mutation in position 184 (NEDFSNFD). The mutations 184 and 1042 were found in almost all samples (Table 1).

\section{Copy number of Pfmdr1}

Pfmdr1 copy number was determined by qPCR. The housekeeping gene Seryl was used as an internal control. 3D7 and Dd2 strains were used as copy number controls, where 3D7 showed one copy and Dd2 two copies of the $P f m d r 1$ gene [30]. Sixty-two samples were analysed, 75\% $(45 / 62)$ of the samples were amplified by qPCR. Onehundred per cent of the samples collected in Esmeraldas county, San Lorenzo county, Carchi and Sucumbíos presented only one copy of Pfmdr1 (Fig. 3).

\section{Live cell imaging}

Ecuadorian P. falciparum isolates were analysed for drug resistance haplotypes at eight different amino acid positions as well as copy number variations of Pfmdr1. These parasites presented two main mutations: Y184F and N1042D and only one copy of the gene. Fluo 4 AM is a fluorochrome and is transported by PfMDR1 from the cytosol to the digestive vacuole (DV) [30, 32, 33]. The use of Fluo 4 AM can help investigate the role of the PfMDR1 transporter. The mutation N1042D has been correlated to reduced transport of Fluo 4 from the cytosol to the DV of the parasite [32]. The parasite
ESM-2013, an isolate from Esmeraldas that presented the mutation N1042D, was cultured with Fluo 4 AM and visualized by confocal microscopy to establish the transport of the fluorochrome. Dd2 strain was used as a positive control that allows the transport of Fluo $4 \mathrm{AM}$ to the DV. The results show that Fluo 4 is not transported to the DV (i.e., Fluor-4 fluorescence does not accumulate) in ESM-2013 parasites (Fig. 4). The ratio of Fluo-4 fluorescence intensity (when calculating DV/ cytosol Fluo-4 fluorescence measured in these compartments) of Dd2 was 6.9. In contrast, ESM-2013 presented a DV/cytosol Fluo-4 fluorescence ratio of 0.50.

\section{In vitro drug sensitivity characterization}

In vitro assays were used to test the sensitivity of Ecuadorian P. falciparum isolates to CQ, MQ, QN, ATM, DHA, and LUMF. ESM-2013 was exposed to antimalarial drugs to establish its $\mathrm{IC}_{50}$. This parasite also presented a genotype associated with $\mathrm{CQ}$ resistance (CVMNT). The parasite ESM-2013 showed an $\mathrm{IC}_{50}$ of $93.71 \mathrm{nM}$ (Table 2). Furthermore, ESM-2013 presented considerably lower $\mathrm{IC}_{50}$ for $\mathrm{MQ}, \mathrm{QN}$, LUMF, ATM, and DHA. ESM-2013 presented $4.94 \mathrm{nM} \mathrm{IC}_{50}$ for MQ, $7.63 \mathrm{nM}$ for QN and $2.60 \mathrm{nM}$ for DHA, indicating a phenotype of sensitivity for those anti-malarial drugs. The current treatment for $P$. falciparum infections in Ecuador is based on ATM and LUMF. This treatment was tested in ESM-2013 and this parasite showed an $\mathrm{IC}_{50}$ of $3.59 \mathrm{nM}$ for LUMF and $1.14 \mathrm{nM}$ for ATM (Table 2). 
Table 1 Drug resistance markers haplotypes by location

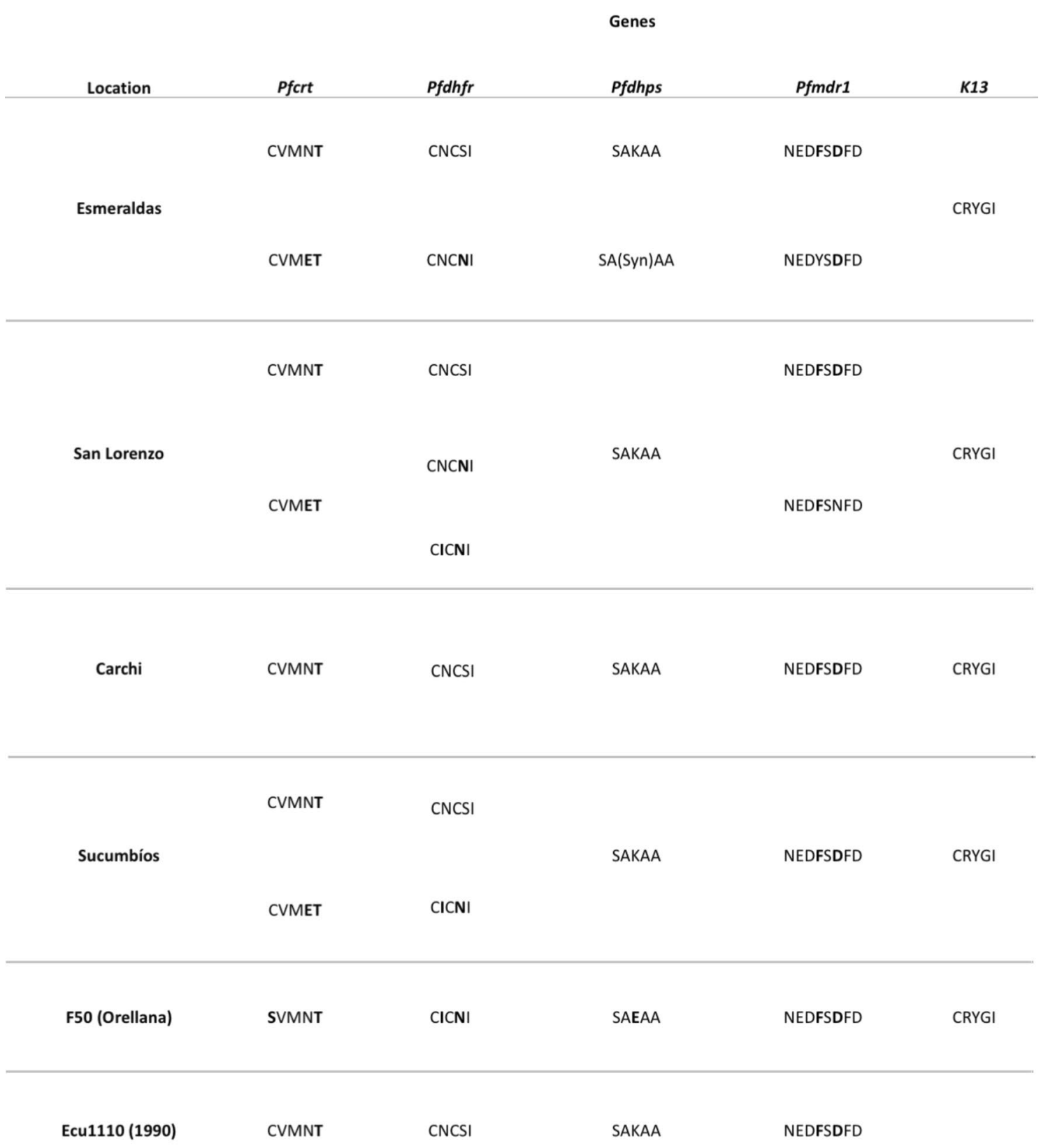

\section{Discussion}

Ecuador has been very successful in reducing the number of malaria cases in the country. It is estimated that more than $99 \%$ prevalence reduction took place from 2000 to 2015 [34]. In this context, understanding drug resistance genotypes and phenotypes of Ecuadorian Plasmodium isolates constitutes crucial information for supporting the National Malaria Programme to achieve elimination of the disease in the country.

Ninety per cent of the samples analysed in this study were collected in Esmeraldas province (Esmeraldas and San Lorenzo counties), where most $P$. falciparum cases are known to occur. In 2016, this province reported 125 P. falciparum cases, roughly $45 \%$ of the total of P. falciparum cases in the country [2]. 


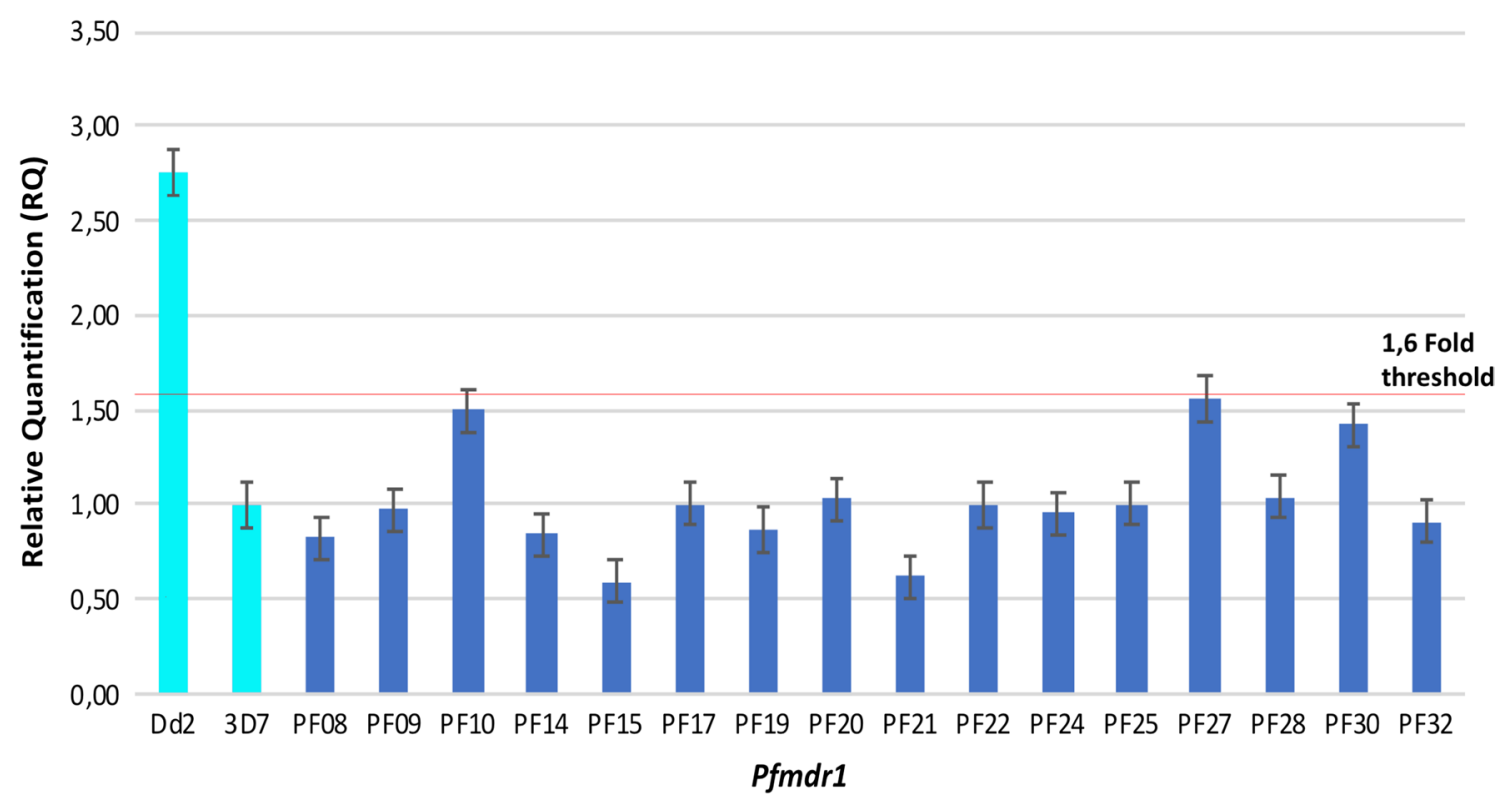

Fig. 3 Pfmdr1 copy number. Dd2 presents two copies and 3D7 one copy. All samples analysed presented one copy of Pfmdr1. Sixteen of the 45 analysed samples are shown. Copy number $>1,6$ was defined as a duplication of pfmdr1 [42]

This study determined the mutations associated with five different genes involved in CQ, SP, MQ, QN, and ART resistance present in Ecuador. The results showed that Ecuadorian parasites presented the CQ resistance haplotypes CVMNT, CVMET and SVMNT in Pfcrt (7276). The genotype CVMNT was found in Esmeraldas, San Lorenzo, Carchi and Sucumbíos while the CVMET genotype was found in San Lorenzo and Sucumbíos, the SVMNT genotype was found in one sample from Orellana. CVMNT and CVMET genotypes have previously been reported in Colombia [8]. CVMNT was reported in the Pacific coast of Peru and the CVMET genotype was reported in the Amazon region of Peru [10] The genotype SVMNT has been found in the Peruvian [10] and Brazilian Amazon [9].

The CVMNT haplotype had been previously reported in a parasite isolate obtained in Esmeraldas in the 1990 [27]. Additionally, this genotype was reported in Ecuador during an outbreak occurred in 2013 [26]. This suggests that this haplotype has been circulating in Ecuador for decades and is still maintained in the country. Griffing and collaborators suggest that parasites carrying this haplotype, were circulating in Colombia and then crossed into Ecuador and later entered Peru [10].

The resistance to CQ is considered fixed in South America, since CVMNT, CVMET and SVMNT are common genotypes in the region [7, 9]. However, in Ecuador, the national treatment regime was changed from CQ in 2004 to artesunate + SP [35] and, more recently, to ATM-LUMF [26]. Plasmodium falciparum parasites circulating in the region continue to have the K76T genotype of Pfcrt. This could be related to continuous drug pressure in the parasite population, since CQ remains the main treatment to control $P$. vivax infections.

The wild type genotype CNCSI for $P f d h f r$ was the main genotype found in all locations sampled in this study (Ecuador, Peru, Colombia, and in the Ecu 1110 isolate) [27]. The genotype with a simple mutation in position 108 (CNCNI) was only found in San Lorenzo county. These genotypes have previously been reported in Ecuador [25], Colombia [8] and Peru [10]. The CICNI resistance genotype was found in San Lorenzo county and Orellana; Peru, Colombia and Brazil have also reported this genotype $[8,9]$.

The Pfdhfr polymorphisms were more diverse in San Lorenzo than in the other Ecuadorian locations. San Lorenzo is located close to the Colombian border suggesting that the migration of parasites from Colombia to Ecuador could be related to the distribution of these mutations. In fact, double and triple mutations in Pfdhfr and resistance to SP have frequently been reported in Colombia [8]. In addition, it has recently been reported using neutral microsatellites that $P$. falciparum populations from San Lorenzo are shared with the south of Colombia [36].

The wild type genotype SAKAA for Pfdhps presented the highest frequency in all studied locations, as well as for Ecu 1110 [27]. Only the sample F50 from Orellana 


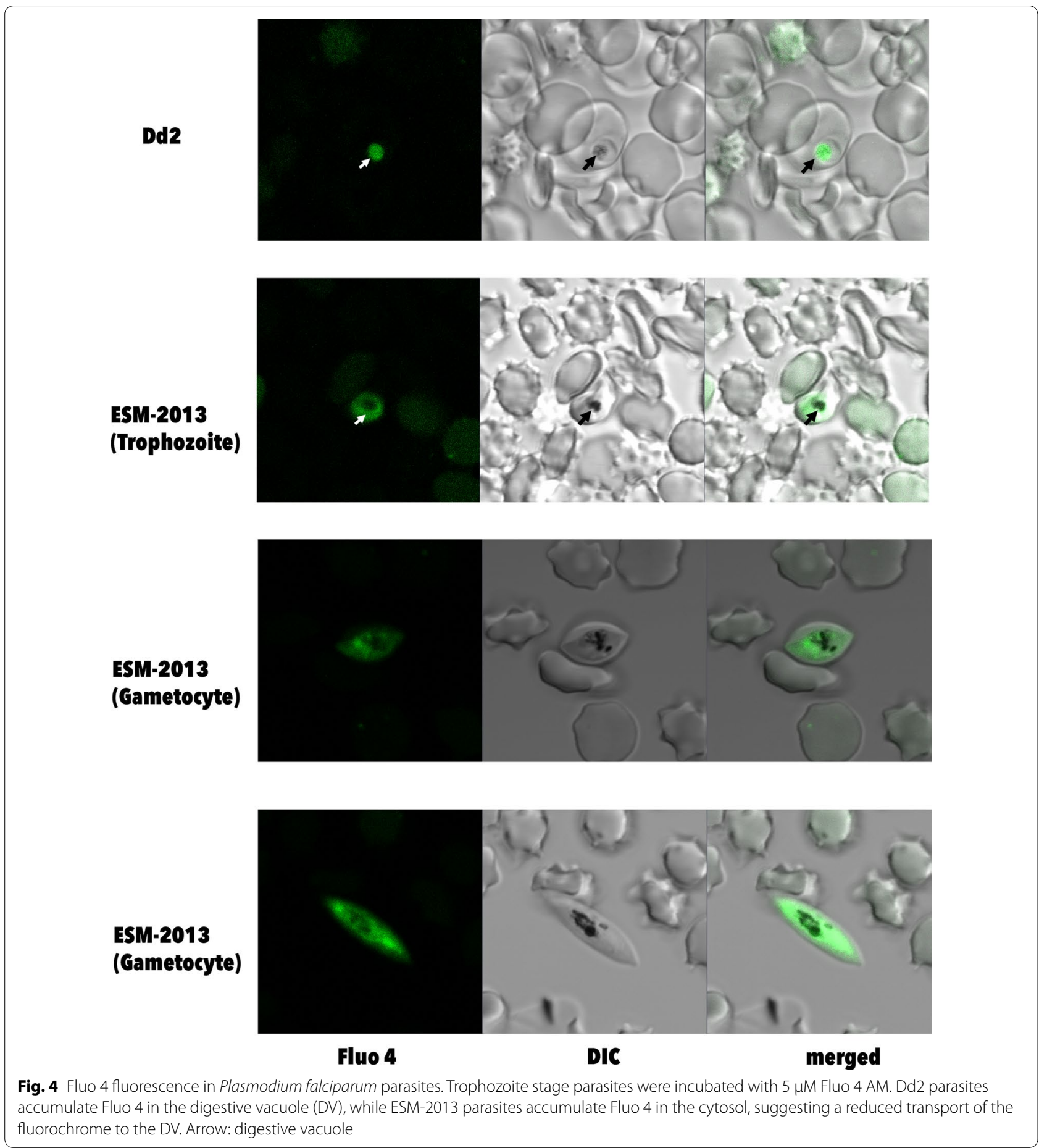

presented mutation in this position (SAEAA). This mutation is common in Brazil [9], Venezuela, Bolivia, and Peru $[8,10]$. The wild type genotype was previously found in samples from Esmeraldas in 2002 and 2013. No mutations in Pfdhps have ever been previously reported in Ecuador $[25,26]$.
Despite of the presence of mutations in $P f d h f r$ and Pfdhps in samples from Ecuador, the high prevalence of wild type genotypes suggests ongoing sensitivity to SP in the country. In 2002, 90\% of samples collected in Esmeraldas presented at least one mutation in $P f d$ hfr (position $108 \mathrm{~N}$ ). In addition, double (positions 
Table 2 IC $_{50}$ values of Plasmodium falciparum ESM-2013 and 3D7

\begin{tabular}{lcc}
\hline Drug & $\begin{array}{l}\text { ESM-2013 } \\
\mathbf{I C}_{\mathbf{5 0}} \text { [nM] }\end{array}$ & $\begin{array}{l}\text { 3D7 } \\
\mathbf{I C}_{\mathbf{5 0}} \text { [nM] }\end{array}$ \\
\hline CQ & $93.71 \pm 33.07$ & $8.36 \pm 5.6$ \\
MQ & $4.94 \pm 0.6$ & $19.3 \pm 2.15$ \\
QN & $7.63 \pm 1.08$ & $23 \pm 1.8$ \\
LUMF & $3.59 \pm 0.4$ & $7.46 \pm 2.6$ \\
ATM & $1.14 \pm 0.7$ & $3.1 \pm 0.8$ \\
DHA & $2.6 \pm 1.4$ & $2.4 \pm 1.3$ \\
\hline
\end{tabular}

$108 \mathbf{N}, 164 \mathrm{~L})$ and triple (51I, 108N, 164L) Pfdhfr mutations were reported [25]. The decrease in the frequency of parasites carrying $P f d h f r$ mutations in Esmeraldas province could be related to the change in treatment from artesunate + SP to ATM-LUMF, which reduced the parasites from drug pressure.

Pfmdr1 codes for a transmembrane P-glycoprotein in the DV of the parasites involved in transport of substrates from the cytosol to the DV. This protein belongs to the adenosine triphosphate-binding cassette transporter family [12]. Two factors have been associated with alteration of function in PfMDR1: mutations present in Pfmdr1 and copy number increase [32]. The mutations N86Y and Y184F are more common in Asia and Africa. In contrast, in South America the mutations S1034C, N1042D and D1246Y are found to be more common [13]. These mutations are associated with multidrug resistance [37].

In Ecuador, the mutations $184 \mathrm{~F}$ and 1042D were found frequently and were present in the majority of samples from an outbreak that occurred in Esmeraldas in 2013 [26]. These double mutants were found in this study in samples from Esmeraldas, San Lorenzo, Carchi and Sucumbíos. Furthermore, this genotype was found in Orellana and previously reported for the Ecu 1110 parasite [27]. The Pfmdr1 polymorphisms (86, 1034, $1042,1246)$ have been associated with resistance to QN, MQ, DHA, and HF [12]. The 184F mutation has not been associated with any specific drug resistance and the mutation 1042D has been linked to MQ and QN resistance [32]. Ecuadorian parasites do not present clinical or in vitro resistance to MQ or $\mathrm{QN}$, suggesting that these drugs can be considered as an alternative to current treatment in the future.

The increase in Pfmdr1 copy number has been associated with $P$. falciparum resistance to $\mathrm{MQ}, \mathrm{QN}$ and ART [14-16]. All Ecuadorian P. falciparum parasites in this study showed one copy of this gene, suggesting that these parasites are sensitive to MQ. Efficacy in vivo studies of artesunate and MQ combination showed that these drugs were an effective treatment in Ecuador in 2000 [38]. In South America, there are reports of modifications in copy number in samples from the Pacific region, Atlantic region and southeast of Colombia (2009-2012), where $32 \%$ of the isolates had Pfmdr1 copy numbers increase to two to five copies [15]. Peru reported single copy number for Pfmdr1 in 2009 in the Amazon region [17].

The mutation 1042D and the increase in copy number have been associated with MQ resistance [32]. MQ forms hydrogen bonds with the residue 1042 of PfMDR1 and the change of $\mathrm{N}$ (asparagine) to $\mathrm{D}$ (acid aspartic) in this position may result in the inhibition of MQ passage through the DV membrane [39]. In order to test this hypothesis, live cell imaging using Fluo 4 AM was performed.

Fluo $4 \mathrm{AM}$ is a fluorochrome that has been used to determine PfMDR1 transport of substrates from the cytosol to the DV of the parasite [32]. The parasites that present N1042 (wild type genotype) show an increased Fluo-4 fluorescence in the DV, showing that the fluorochrome is readily transported into this compartment [30, 32, 33]. In contrast, the parasites with 1042D (mutated) PfMDR1 show no increase in Fluo-4 fluorescence in the DV (there is rather an increase in fluorescence in the cytosol of the parasites), suggesting that the fluorochrome is not transported into the DV [30,32, 33].

The mutation N1042D was found in most Ecuadorian P. falciparum isolates tested in this study, including ESM2013. The Fluo 4 AM assay was performed in the isolate ESM-2013 from Esmeraldas to confirm that this mutation inhibited the transport of this marker. The fluorescence intensity of Fluo-4 in the DV of Dd2 (N1042) was higher than in ESM-2013. The Ecuadorian P. falciparum presented the mutation 1042D and inhibited the transport of Fluo 4 into the DV and did not present in vitro resistance to $M Q$. This suggests that resistance to $M Q$ can be related to the synergy between polymorphisms and increase in copy number of Pfmdr1. The results showed that PfMDR1 of ESM-2013 is not completely functional, since there was inhibition of Fluo 4 transport. These results are not directly related with the current treatment for P. falciparum in Ecuador but should be considered in case a treatment change is planned.

Plasmodium falciparum resistance to ART has been reported in Southeast Asian countries, particularly in the Grand Mekong area (China, Vietnam, Cambodia, Thailand, Myanmar). ART resistance has been associated with mutations in $k 13$ [18, 19, 21, 40]. All Ecuadorian samples presented the wild type CRYGI (positions 476, 493, 539, 543, 580) genotype for this gene. Similarly, recent studies in Brazilian, Peruvian 
and Colombian isolates have shown a lack of $k 13$ mutations and the ACT treatment appears to be effective in these South American regions. The mutation C580Y (associated with ART susceptibility in Southeast Asia) was found in 5\% of P. falciparum isolates from Guyana, even though ART showed 100\% efficacy [20]. Although no ATM resistance mutations were found in the studied samples, the spread of other mutations related to ART resistance cannot be ruled out.

Drug resistance phenotypes can also be characterized by in vitro assays. In this study, in vitro assays were used to associate drug susceptibility phenotypes to drug-resistant genotypes. In vitro studies are used to monitor the drug susceptibility of $P$. falciparum and help guide the drug policy in each country. These studies can give a better idea of how the interaction between the parasite and the drug occur and can help establish parasite sensitivity.

Ecuadorian parasites were cultured and exposed to common anti-malarial drugs to establish their drug susceptibility. The ESM-2013 parasite showed an $\mathrm{IC}_{50}$ of $93.7 \mathrm{nM}$, confirming a CQ-resistant phenotype in Ecuadorian $P$. falciparum isolates having the mutation K76T. The in vitro resistance to CQ in Ecuadorian parasites has been previously reported in Ecu1110. It presented an $\mathrm{IC}_{50}>90.9 \mathrm{nM}$ [27] in comparison with 3D7 that showed an $\mathrm{IC}_{50}<10 \mathrm{nM}$. Other in vitro studies have shown resistance to CQ in Colombia, where $90 \%$ of parasites analysed presented $\mathrm{IC}_{50}>100 \mathrm{nM}$ [22]. CQ resistance has been present in Ecuador since the 1980 s, suggesting that the resistance to CQ is fixed in Ecuadorian P. falciparum parasites. ESM-2013 showed in vitro sensitivity to QN, MQ, DHA, LUMF, and ATM; similarly, Colombian samples had in vitro sensitivity to DHA, LUMF and ATM [15].

The current treatment for P. falciparum in Ecuador is ATM-LUMF. The results of this study suggest that this treatment continues to be effective in the country, as well as in the rest of Latin America where there is no reported resistance to ACT treatment [5]. It is important to note that, even though Ecuadorian parasites have CQ-resistant genotype and phenotype and present mutations in Pfdhfr and Pfmdr1, they have the same resistance profile as Ecu 1110, an isolate collected in 1990 [26]. These results suggest that the mutations in drug resistance genes have been maintained for almost 30 years in spite of a lack of selective pressure. This could be explained by a fixation of drug resistant mutations and the presence of parasites as asymptomatic reservoirs [41].

\section{Conclusion}

This study shows that Ecuadorian P. falciparum had chloroquine-resistant genotype and phenotype, but were sensitive to sulfadoxine, pyrimethamine, artemether, lumefantrine, quinine, mefloquine, and dihydroartemisinin, indicating that the status of anti-malarial resistance during 2013-2015 remained effective. Nevertheless, this study will need to be completed with samples from the Amazon region to clarify the situation of resistance in all endemic areas of Ecuador. This work shows the importance of combining molecular and in vitro studies as surveillance tools to aid malaria elimination in the country. Early detection of resistance to the current drug treatment used for $P$. falciparum infections is essential to hinder outbreaks. This study presents the status of antimalarial resistance in Ecuador and informs malaria elimination campaigns in the country.

\section{Supplementary information}

Supplementary information accompanies this paper at https://doi. org/10.1186/s12936-019-3044-z.

Additional file 1: Table S1. Primers and conditions used for amplification of drug resistance markers.

\section{Acknowledgements}

We would like to thank the personnel of the Ecuadorian Ministry of Public Health taking part in the sample collection and all the patients participating in this study.

\section{Authors' contributions}

FES and PR conceived and designed the study; GV, PR and FES wrote the manuscript; GV, CAV and PR performed the experiments; GV, FES and PR analyzed the data; JV and LEC performed field work. All authors read and approved the final manuscript.

\section{Funding}

Pontificia Universidad Católica de Ecuador (Grants L13058, L13248, M13416 and N13416) and Government of Canada (Global Affairs, Emerging Leaders in the Americas Program).

\section{Availability of data and materials}

The datasets used and/or analyzed during the current study are available from the corresponding author on reasonable request

\section{Ethics approval and consent to participate}

The study protocol was approved by the Ethical Review Committees of Pontificia Universidad Católica del Ecuador (approvals \#: CBE-016-2013 and 20-11-14-01).

Consent for publication

Not applicable

Competing interests

The authors declare that they have no competing interests.

\section{Author details}

${ }^{1}$ Centro de Investigación para la Salud en América Latina, Facultad de Ciencias Exactas y Naturales, Pontificia Universidad Católica del Ecuador, Av. 12 de Octubre 1076, Apartado, 17-01-2184 Quito, Ecuador. ${ }^{2}$ Ministerio de Salud Pública, Guayaquil, Ecuador. ${ }^{3}$ Institute of Parasitology, McGill University, Montreal, Canada. 
Received: 11 August 2019 Accepted: 28 November 2019

Published online: 10 December 2019

\section{References}

1. World Health Organization. World malaria report. Geneva: World Health Organization; 2016.

2. MSP. Gaceta Epidemiológica Semanal No. 52: Ministerio de Salud Pública del Ecuador. Quito: MSP; 2017.

3. PAHO. Report on the situation of malaria in the Americas, 2000-2015. Geneva: World Health Organization; 2016.

4. WHO. World malaria report. Geneva: World Health Organization; 2018

5. Recht J, Siqueira AM, Monteiro WM, Herrera SM, Herrera S, Lacerda MVG. Malaria in Brazil, Colombia, Peru and Venezuela. Current challenges in malaria control and elimination. Malar J. 2017;16:273.

6. Fidock DA, Nomura T, Talley AK, Cooper RA, Dzekunov SM, Ferdig MT, et al. Mutations in the $P$. falciparum digestive vacuole transmembrane protein PfCRT and evidence for their role in chloroquine resistance. Mol Cell. 2000;6:861-71.

7. Pelleau S, Moss EL, Dhingra SK, Volney B, Casteras J, Gabryszewski SJ, et al. Adaptive evolution of malaria parasites in French Guiana: reversal of chloroquine resistance by acquisition of a mutation in pfert. Proc Natl Acad Sci USA. 2015;112:11672-7.

8. Cortese JF, Caraballo A, Contreras CE, Plowe CV. Origin and dissemination of Plasmodium falciparum drug-resistance mutations in South America. J Infect Dis. 2002;186:999-1006.

9. Corredor V, Murillo C, Echeverry DF, Benavides J, Pearce RJ, Roper C, et al. Origin and dissemination across the Colombian Andes mountain range of sulfadoxine-pyrimethamine resistance in Plasmodium falciparum. Antimicrob Agents Chemother. 2010;54:3121-5.

10. Griffing SM, Mixson-Hayden T, Sridaran S, Alam MT, McCollum AM, Cabezas C, et al. South American Plasmodium falciparum after the malaria eradication era: clonal population expansion and survival of the fittest hybrids. PLOS ONE. 2011;6:e23486.

11. Sridaran S, Rodriguez B, Mercedes Soto A, Macedo De Oliveira A, Udhayakumar V. Molecular analysis of chloroquine and sulfadoxinepyrimethamine resistance-associated alleles in Plasmodium falciparum isolates from Nicaragua. Am J Trop Med Hyg. 2014;90:840-5.

12. Price RN, Uhlemann A-C, Brockman A, McGready R, Ashley E, Phaipun $L$, et al. Mefloquine resistance in Plasmodium falciparum and increased pfmdr1 gene copy number. Lancet. 2004;364:438-47.

13. Veiga MI, Dhingra SK, Henrich PP, Straimer J, Gnädig N, Uhlemann A-C, et al. Globally prevalent PfMDR1 mutations modulate Plasmodium falciparum susceptibility to artemisinin-based combination therapies. Nat Commun. 2016:7:11553.

14. Ferreira ID, do Rosário VE, Cravo PV. Real-time quantitative PCR with SYBR Green I detection for estimating copy numbers of nine drug resistance candidate genes in Plasmodium falciparum. Malar J. 2006;5:1.

15. Aponte S, Patricia Guerra Á, Álvarez-Larrotta C, Bernal S, Restrepo C, González C, et al. Baseline in vivo, ex vivo and molecular responses of Plasmodium falciparum to artemether and lumefantrine in three endemic zones for malaria in Colombia. Trans R Soc Trop Med Hyg. 2017;111:71-80.

16. Costa GL, Amaral LC, Fontes CJF, Carvalho LH, de Brito CFA, de Sousa TN. Assessment of copy number variation in genes related to drug resistance in Plasmodium vivax and Plasmodium falciparum isolates from the Brazilian Amazon and a systematic review of the literature. Malar J. 2017;16:152.

17. Bacon DJ, McCollum AM, Griffing SM, Salas C, Soberon V, Santolalla $M$, et al. Dynamics of malaria drug resistance patterns in the Amazon Basin region following changes in Peruvian national treatment policy for uncomplicated malaria. Antimicrob Agents Chemother. 2009;53:2042-51.

18. Straimer J, Gnädig NF, Witkowski B, Amaratunga C, Duru V, Ramadani $\mathrm{AP}$, et al. K13-propeller mutations confer artemisinin resistance in Plasmodium falciparum clinical isolates. Science. 2015;347:428-31.

19. Cheeseman IH, Miller BA, Nair S, Nkhoma S, Tan A, Tan JC, et al. A major genome region underlying artemisinin resistance in malaria. Science. 2012;336:79-82.
20. Chenet SM, Schneider KA, Villegas L, Escalante AA. Local population structure of Plasmodium: impact on malaria control and elimination. Malar J. 2012;11:412.

21. Chenet SM, Okoth SA, Kelley J, Lucchi N, Huber CS, Vreden S, et al. Molecular profile of malaria drug resistance markers of Plasmodium falciparum in Suriname. Antimicrob Agents Chemother. 2017:61:e02655-16.

22. Aponte SL, Díaz G, Pava Z, Echeverry DF, Ibarguen D, Rios M, et al. Sentinel network for monitoring in vitro susceptibility of Plasmodium falciparum to antimalarial drugs in Colombia: a proof of concept. Mem Inst Oswaldo Cruz. 2011;106:123-9.

23. Segurado A, Di Santi S, Shiroma M. In vivo and in vitro Plasmodium falciparum resistance to chloroquine, amodiaquine and quinine in the Brazilian Amazon. Rev Inst Med Trop São Paulo. 1997;39:85-90.

24. White J, Mascarenhas A, Pereira L, Dash R, Walke JT, Gawas P, et al. In vitro adaptation of Plasmodium falciparum reveal variations in cultivability. Malar J. 2016;15:33.

25. Arróspide N, Hijar-Guerra G, de Mora D, Diaz-Cortéz CE, Veloz-Perez $\mathrm{R}$, Gutierrez S, et al. Alelos mutantes asociados a la resistencia a cloroquina y sulfadoxina-pirimetamina en Plasmodium falciparum de las fronteras Ecuador-Perú y Ecuador-Colombia. Rev Peru Med Exp Salud Pública. 2014;31:282-7.

26. Sáenz FE, Morton LC, Okoth SA, Valenzuela G, Vera-Arias CA, Vélez-Álvarez $\mathrm{E}$, et al. Clonal population expansion in an outbreak of Plasmodium falciparum on the northwest coast of Ecuador. Malar J. 2015;14:497.

27. Sá JM, Twu O, Hayton K, Reyes S, Fay MP, Ringwald P, et al. Geographic patterns of Plasmodium falciparum drug resistance distinguished by differential responses to amodiaquine and chloroquine. Proc Natl Acad Sci USA. 2009:106:18883-9.

28. Moll K, Kaneko A, Scherf A, Wahlgren M. Methods in malaria research. 6th ed. Manassas: MR4/ATCC; 2013

29. Snounou G. Detection and identification of the four malaria parasite species infecting humans by PCR amplification. Methods Mol Biol. 1996;50:263-91.

30. Friedrich O, Reiling SJ, Wunderlich J, Rohrbach P. Assessment of Plasmodium falciparum PfMDR1 transport rates using Fluo-4. J Cell Mol Med. 2014;18:1851-62.

31. Schuster FL. Cultivation of Plasmodium spp. Clin Microbiol Rev. 2002;15:355-64.

32. Reiling SJ, Rohrbach P. Monitoring PfMDR1 transport in Plasmodium falciparum. Malar J. 2015;14:270.

33. Rohrbach P, Sanchez CP, Hayton K, Friedrich O, Patel J, Sidhu ABS, et al. Genetic linkage of pfmdr1 with food vacuolar solute import in Plasmodium falciparum. EMBO J. 2006;25:3000-11.

34. MSP. Situación de la malaria en el Ecuador. Gaceta Ministerio de Salud Pública del Ecuador. Quito, 2013.

35. WHO. Country epidemiological profile: Ecuador. Geneva: World Health Organization; 2018.

36. Vera-Arias CA, Castro LE, Gómez-Obando J, Sáenz FE. Diverse origin of Plasmodium falciparum in northwest Ecuador. Malar J. 2019;18:251.

37. Li J, Chen J, Xie D, Monte-Nguba S, Eyi JUM, Matesa RA, et al. High prevalence of pfmdr1 N86Y and Y184F mutations in Plasmodium falciparum isolates from Bioko island, Equatorial Guinea. Pathog Glob Health. 2014;108:339-43.

38. Gomez LEA, Jurado MH, Cambon N. Randomised efficacy and safety study of two 3-day artesunate rectal capsule/mefloquine regimens versus artesunate alone for uncomplicated malaria in Ecuadorian children. Acta Trop. 2003:89:47-53.

39. Patel SK, George L-B, Prasanth Kumar S, Highland HN, Jasrai YT, Pandya $\mathrm{HA}$, et al. A Computational approach towards the understanding of Plasmodium falciparum multidrug resistance protein 1. ISRN Bioinforma. 2013;2013:437168.

40. Sidhu ABS, Uhlemann A-C, Valderramos SG, Valderramos J-C, Krishna S, Fidock DA. Decreasing pfmdr1 copy number in Plasmodium falciparum malaria heightens susceptibility to mefloquine, lumefantrine, halofantrine, quinine, and artemisinin. J Infect Dis. 2006;194:528-35.

41. Sáenz FE, Arévalo-Cortés A, Valenzuela G, Vallejo AF, Castellanos A, Poveda-Loayza AC, et al. Malaria epidemiology in low-endemicity areas of the northern coast of Ecuador: high prevalence of asymptomatic infections. Malar J. 2017;16:300. 
42. Ladeia-Andrade S, de Melo GNP, de Souza-Lima RD, Salla LC, Bastos MS, Rodrigues PT, et al. No clinical or molecular evidence of Plasmodium falciparum resistance to artesunate-mefloquine in Northwestern Brazil. Am J Trop Med Hyg. 2016;95:148-54.

\section{Publisher's Note}

Springer Nature remains neutral with regard to jurisdictional claims in published maps and institutional affiliations.
Ready to submit your research? Choose BMC and benefit from:

- fast, convenient online submission

- thorough peer review by experienced researchers in your field

- rapid publication on acceptance

- support for research data, including large and complex data types

- gold Open Access which fosters wider collaboration and increased citations

- maximum visibility for your research: over $100 \mathrm{M}$ website views per year

At BMC, research is always in progress.

Learn more biomedcentral.com/submissions 